\title{
OSCILLATION THEOREMS FOR SOLUTIONS OF HYPERBOLIC EQUATIONS
}

\author{
CHARLES KAHANE
}

ABstract. It is shown that solutions of hyperbolic equations in cylindrical space time domains which vanish on the lateral boundary of the cylinder must have arbitrarily large zeros in the interior of the cylinder. In case the coefficients of the equation are time independent the solutions will have arbitrarily large zeros on any interior line of the cylinder.

This paper concerns the oscillations of solutions of hyperbolic equations. More specifically, we shall consider solutions of the equation

$$
u_{\| t}=\sum_{j, k} \frac{\partial}{\partial x_{k}}\left(a_{j k}(x, t) \frac{\partial u}{\partial x_{j}}\right)-c(x, t) u
$$

in some space-time cylinder $\Omega \times(0, \infty)$, where we assume that the operator on the right is uniformly elliptic:

$$
\sum_{j . k} a_{j k}(x, t) \xi_{j} \xi_{k} \geqq \mu|\xi|^{2} \quad(\mu>0)
$$

and that $c(x, t) \geqq 0$ in $\Omega \times(0, \infty)$. We shall further assume that the coefficients $a_{j k}$ are in $C^{1}(\bar{\Omega} \times(0, \infty))$ and that their derivatives are uniformly bounded in $\bar{\Omega} \times(0, \infty)$; while $c(x, t)$ will be assumed continuous in $\bar{\Omega} \times(0, \infty)$. We will first want to consider classical solutions of (1) with well-defined boundary values on $\partial \Omega \times(0, \infty)$ and we will take this to mean a function $u(x, t)$ in $C^{2}(\bar{\Omega} \times(0, \infty))$ which satisfies $(1)$ in $\Omega \times(0, \infty)$. Concerning $\Omega$ we assume it to be a bounded domain in $E^{n}$ with $\partial \Omega$ a sufficiently smooth $n-1$ dimensional manifold having a well-defined exterior normal at every point. This being understood our first result is

THEOREM 1. Let $u(x, t)$ be a solution of $(1)$ in $\Omega \times(0, \infty)$ which vanishes on $\partial \Omega \times(0, \infty)$. Then for any $\tau>0, u$ must have a zero in $\Omega \times[\tau, \infty)$.

REMARK. A result of this type has been obtained by K. Kreith [3] for the case of one space variable $x_{1}$ with the operator on the right of (1) having the form $\partial^{2} / \partial x_{1}^{2}-\kappa\left(x_{1}, t\right)$. His method extends to second order elliptic operators in higher dimensions provided that the principal part

Received by the editors October 12, 1972 and, in revised form, January 19, 1973. AMS (MOS) subject classifications (1970). Primary 35B05.

Key words and phrases. Oscillation, hyperbolic equations. 
admit separation of variables. No such restriction is placed on the elliptic operators considered in Theorem 1, so that it applies to a more general class of equations.

Proof. Assume on the contrary, that $u$ never vanishes in $\Omega \times[\tau, \infty)$ and for definiteness suppose that $u$ is positive in this set. We shall obtain a contradiction by showing that for some suitable positive $\phi(x) \in C^{2}(\bar{\Omega})$, the integrals

$$
I(t)=\int_{\Omega} u(x, t) \phi(x) d x
$$

cannot be positive for all $t \geqq \tau$.

Our choice of $\phi$ will be guided by the requirement that $M(\phi) \leqq-1$ in $\Omega$ for all elliptic operators

$$
M=\sum_{j, k} \alpha_{j k}(x) \frac{\partial^{2}}{\partial x_{j} \partial x_{k}}+\sum_{j} \beta_{j}(x) \frac{\partial}{\partial x_{j}}-\kappa(x)
$$

whose coefficients satisfy the conditions

(4) $\sum_{j, k} \alpha_{j k}(x) \xi_{j} \xi_{k} \geqq \mu|\xi|^{2} \quad(\mu>0), \quad\left(\sum_{j} \beta_{j}^{2}(x)\right)^{1 / 2} \leqq \beta \quad$ and $\quad \kappa(x) \geqq 0$

in $\Omega$. To construct such a $\phi$, let $\rho$ be the radius of an open sphere centred at the origin which contains $\bar{\Omega}$ and let $f(r)$ denote the solution of $\mu f^{\prime \prime}(r)-$ $\beta f^{\prime}(r)=-1$ on $[0, \rho]$ with $f(\rho)=f^{\prime}(0)=0$; that is, the function

$$
f(r)=\left(1 / \mu \gamma^{2}\right)\left(e^{\gamma \rho}-\gamma \rho\right)-\left(1 / \mu \gamma^{2}\right)\left(e^{\gamma r}-\gamma r\right),
$$

where $\gamma=\beta \mu^{-1}$. We now take $\phi(x)=f(|x|)$. It is easily verified that $\phi(x) \in$ $C^{2}\left(E^{n}\right)$, is positive for $|x|<\rho$ and hence positive for $x \in \bar{\Omega}$. Furthermore,

$$
\begin{aligned}
M(\phi)= & {\left[f^{\prime \prime}(|x|)-\frac{f^{\prime}(|x|)}{|x|}\right] \sum_{j, k} \alpha_{j k}(x) \frac{x_{j}}{|x|} \frac{x_{k}}{|x|} } \\
& +\frac{f^{\prime}(|x|)}{|x|} \sum_{j} \alpha_{j j}(x)+f^{\prime}(|x|) \sum_{j} \beta_{j}(x) \frac{x_{j}}{|x|}-f(|x|) \kappa(x)
\end{aligned}
$$

so that in view of $f(r) \geqq 0, f^{\prime}(r) \leqq 0, f^{\prime \prime}(r)-f^{\prime}(r) / r \leqq 0$ when $r \in[0, \rho]$, and the conditions (4)

$$
\begin{aligned}
M(\phi) & \leqq\left[f^{\prime \prime}(|x|)-\frac{f^{\prime}(|x|)}{|x|}\right] \mu+\frac{f^{\prime}(|x|)}{|x|} \mu+f^{\prime}(|x|)\left(\sum_{j} \beta_{j}^{2}(x)\right)^{1 / 2} \\
& \leqq \mu f^{\prime \prime}(|x|)-\beta f^{\prime}(|x|)=-1
\end{aligned}
$$

in $\Omega$. Hence for sufficiently small positive $\varepsilon$, e.g. $\varepsilon=\left[\sup _{\Omega} \phi\right]^{-1 / 2}$, it will then follow that

$$
M(\phi)+\varepsilon^{2} \phi \leqq 0
$$


in $\Omega$ for all $M$ satisfying the conditions (4). We note that the construction of $\phi$ is closely related to the construction of a "strong barrier" (cf. [1]).

We now multiply equation (1) by $\phi$ and integrate over $\Omega$ :

$$
\int_{\Omega} u_{t t} \phi d x=\int_{\Omega} \operatorname{Lu} \phi d x
$$

where $L$ denotes the operator on the right on (1), i.e.

$$
L=\sum_{j, k} \frac{\partial}{\partial x_{k}}\left(a_{j k}(x, t) \frac{\partial}{\partial x_{j}}\right)-c(x, t) .
$$

Integrating by parts we then obtain, since $u$ vanishes on $\partial \Omega \times(0, \infty)$,

$$
\int_{\Omega} u_{t t} \phi d x-\int_{\Omega} u L^{*} \phi d x=\int_{\Omega} \phi \frac{\partial u}{\partial v} d \sigma
$$

here $L^{*}=\sum_{j, k}\left(\partial / \partial x_{j}\right)\left(a_{j k}(x, t) \partial / \partial x_{k}\right)-c(x, t)$ is the operator adjoint to $L$, $d \sigma$ denotes the element of area on $\partial \Omega$, and at any point $x$ of $\partial \Omega, \partial / \partial v$ denotes the directional derivative

$$
\frac{\partial}{\partial v}=\sum_{j, k} a_{j k}(x, t) \gamma_{k} \frac{\partial}{\partial x_{j}},
$$

$\gamma_{k}$ being the $k$ th component of the exterior unit normal to $\partial \Omega$ at $x$. Because of the ellipticity assumption (2), $\partial / \partial v$ is outwardly directed from $\partial \Omega$. Since $u>0$ in $\Omega$ and vanishes on $\partial \Omega$ when $t \geqq \tau$, it follows that any exterior derivative on $\partial \Omega$ such as $\partial u / \partial v$ must be nonpositive for $t \geqq \tau$. Therefore, (6) leads to

$$
\int_{\Omega} u_{t t} \phi d x-\int_{\Omega} u L^{*} \phi d x \leqq 0
$$

for $t \geqq \tau$. Now for fixed but arbitrary $t>0, L^{*}$ is an elliptic operator satsifying the conditions (4). Hence, by (5), $-L^{*} \phi \geqq \varepsilon^{2} \phi$; and we conclude that $I(t)=\int_{\Omega} u(x, t) \phi(x) d x$ satisfies the differential inequality

$$
I^{\prime \prime}(t)+\varepsilon^{2} I(t) \leqq 0 \quad \text { for } t \geqq \tau .
$$

To see that this inequality is incompatible with the positivity of $I(t)$ for $t \geqq \tau$ we multiply it through by $\sin \varepsilon(t-\tau)$ and then integrate over the interval $[\tau, \tau+\pi / \varepsilon]$ on which this factor is nonnegative:

$$
\int_{\tau}^{\tau+\pi / \varepsilon} I^{\prime \prime}(t) \sin \varepsilon(t-\tau) d t+\varepsilon^{2} \int_{\tau}^{\tau+\pi / \varepsilon} I(t) \sin \varepsilon(t-\tau) d t \leqq 0 .
$$

Integrating by parts this becomes

$$
\varepsilon[I(\tau+\pi / \varepsilon)+I(\tau)] \leqq 0,
$$


and thus $I(t)$ cannot be positive for $t \geqq \tau$. This completes the proof of Theorem 1.

In case the coefficients in (1) are independent of $t$ a stronger result holds. It will be more convenient to formulate this result in terms of generalized solutions. Accordingly, let $H_{0}^{1}(\Omega)$ denote the closure of $C_{0}^{\infty}(\Omega){ }^{1}$ under the norm

$$
\|v\|=\left(\int_{\Omega} \sum v_{x_{j}}^{2}(x) d x\right)^{1 / 2}
$$

we will regard the elements of $H_{0}^{1}(\Omega)$ as vanishing in a generalized sense on $\partial \Omega$. Suppose now that for fixed $t \in(0, \infty), u(x, t) \in H_{0}^{1}(\Omega)$ and that as a function of $t, u(x, t)$ is continuous in the $H_{0}^{1}(\Omega)$ norm and continuously differentiable in the $L_{2}(\Omega)$ norm, i.e.

$$
\left\|\frac{u(x, t+\Delta t)-u(x, t)}{\Delta t}-u_{t}(x, t)\right\|_{L_{2}(\Omega)} \rightarrow 0
$$

as $\Delta t \rightarrow 0$, with $u_{t}(x, t)$ continuous as a function of $t$ in the $L_{2}(\Omega)$ norm. Such a function will be said to be a generalized solution of the equation

$$
u_{t t}=\sum_{j, k} \frac{\partial}{\partial x_{k}}\left(a_{j k}(x) \frac{\partial u}{\partial x_{j}}\right)-c(x) u
$$

in $\Omega \times(0, \infty)$ if the identity

$$
\iint_{\Omega} u_{t} \psi_{t} d x d t=\iint_{\Omega}\left[\sum_{j, k} a_{j k} \frac{\partial u}{\partial x_{j}} \frac{\partial \psi}{\partial x_{k}}+c u \psi\right] d x d t
$$

holds for any $\psi(x, t) \in C_{0}^{\infty}(\Omega \times(0, \infty))$.

Our assumptions on the coefficients in (7) are uniform ellipticity: $\sum_{j, k} a_{j k}(x) \xi_{j} \xi_{k} \geqq \mu|\xi|^{2}$, symmetry: $a_{j k}(x)=a_{k j}(x)$, and $c(x) \geqq 0$ in $\Omega$; as to their smoothness, we suppose that $a_{j k}(x) \in C^{2}(\bar{\Omega})$ and $c(x) \in C^{1}(\bar{\Omega})$. Provided that $\partial \Omega$ is suitably smooth, these assumptions are sufficient to guarantee (cf. [2]) the existence of a complete orthogonal set of $C^{2}(\Omega)$ eigenfunctions for the problem $L \phi+\lambda \dot{\phi}=0$ in $\Omega, \phi=0$ on $\partial \Omega, L$ being the operator on the right of (7); the eigenvalues forming a sequence of real positive numbers tending to $\infty$. Furthermore, any generalized solution $u(x, t)$ of (7) can then be represented in terms of a normalized set of eigenfunctions $\left\{\phi_{j}(x)\right\}$ and corresponding eigenvalues $\lambda_{j}$ in a series of the form

$$
u(x, t)=\sum_{j}\left[\alpha_{j} \cos \lambda_{j}^{1 / 2}\left(t-t_{0}\right)+\beta_{j} \sin \lambda_{j}^{1 / 2}\left(t-t_{0}\right)\right] \phi_{j}(x),
$$

${ }^{1} C_{0}^{\infty}(S)$ denotes the set of infinitely differentiable functions with compact support contained in the open set $S$. 
where $t_{0}$ is any positive number, with the series converging in $L_{2}(\Omega)$ for each $t \in(0, \infty)$ so that

$$
\sum_{j}\left(\alpha_{j}^{2}+\beta_{j}^{2}\right)<\infty
$$

We are now prepared to state our second result.

THEOREM 2. Let $u(x, t)$ be a generalized soiution of $(7)$ in $\Omega \times(0, \infty)$ (and thus "vanishing" on $\partial \Omega \times(0, \infty)$ in the sense of being an element in $\left.H_{0}^{1}(\Omega)\right)$. Assume in addition that $u(x, t)$ is continuous in $\Omega \times(0, \infty)$. Then for each fixed $x \in \Omega, u(x, t)$ vanishes for a sequence of values of $t$ which approach $\infty$.

Remark. Conditions ensuring the continuity of generalized solutions of (7) are described in [2].

Proof. In view of (9), the series (8) converges in $L_{2}(\Omega)$ uniformly with respect to $t>0$. Accordingly, we may integrate it termwise and this yields

$$
\begin{aligned}
\int_{t_{0}}^{t} u(x, \tau) d \tau & \\
= & \sum_{j} \lambda_{j}^{-1 / 2}\left[\alpha_{j} \sin \lambda_{j}^{1 / 2}\left(t-t_{0}\right)-\beta_{j} \cos \lambda_{j}^{1 / 2}\left(t-t_{0}\right)\right] \phi_{j}(x)+c_{0}(x)
\end{aligned}
$$

for $t>0$, where $c_{0}(x)=\sum_{j} \lambda_{j}^{-1 / 2} \beta_{j} \phi_{j}(x)$ is a function in $L_{2}(\Omega)$. Repeating this procedure, say, altogether $2 m$ times, we obtain

$$
\begin{aligned}
& \int_{t_{0}}^{t} \frac{(t-\tau)^{2 m-1}}{(2 m-1) !} u(x, \tau) d \tau \\
& =\sum_{j}(-1)^{m} \lambda_{j}^{-m}\left[\alpha_{j} \cos \lambda_{j}^{1 / 2}\left(t-t_{0}\right)+\beta_{j} \sin \lambda_{j}^{1 / 2}\left(t-t_{0}\right)\right] \phi_{j}(x) \\
& \quad+\sum_{k=0}^{2 m-1} c_{k}(x) \frac{\left(t-t_{0}\right)^{2 m-1-k}}{(2 m-1-k) !}
\end{aligned}
$$

for $t>0$, with the $c_{k}(x)$ 's certain elements in $L_{2}(\Omega)$.

Now if $m$ is sufficiently large the series $\sum_{j} \lambda_{j}^{-2 m} \phi_{j}^{2}(x)$ converges pointwise uniformly in $\Omega$ [2, Lemma 1, p. 111]. It then follows by (9) and Schwarz's inequality that the series

$$
S_{m}(x, t)=\sum_{j}(-1)^{m} \lambda_{j}^{-m}\left[\alpha_{j} \cos \lambda_{j}^{1 / 2}\left(t-t_{0}\right)+\beta_{j} \sin \lambda_{j}^{1 / 2}\left(t-t_{0}\right)\right] \phi_{j}(x)
$$

converges uniformly with respect to the $L_{\alpha}$ norm in $\Omega \times(-\infty,+\infty)$ for $m$ suitably large. Thus, $S_{m}(x, t)$ is continuous in $\Omega \times(0, \infty)$. Since the same is true of

$$
\int_{t_{0}}^{t} \frac{(t-\tau)^{2 m-1}}{(2 m-1) !} u(x, \tau) d \tau
$$


(in view of the assumed continuity of $u(x, \tau)$ ), each of the $L_{2}(\Omega)$ functions $c_{k}(x)$ appearing on the right of (10) is actually a continuous function of $x$ and hence well-defined everywhere in $\Omega$. There is, therefore, no difficulty in concluding that for every fixed value of $x \in \Omega$, the function

$$
\sum_{k=0}^{2 m-1} c_{k}(x) \frac{\left(t-t_{0}\right)^{2 m-1-k}}{(2 m-1-k) !}
$$

has derivatives of all orders with respect to $t$. Accordingly, from (10), we see that for $x \in \Omega, S_{m}(x, t)$ has derivatives up to order $2 m$ with respect to $t$, and that

$$
\left(\frac{d}{d t}\right)^{2 m} S_{m}(x, t)=u(x, t) \text { in } \Omega \times(0, \infty) .
$$

Next, as a further consequence of the uniform convergence in $\Omega \times(-\infty$, $+\infty)$ of the series (11), its sum $S_{m}(x, t)$ represents an almost periodic function in $t$ for each fixed $x \in \Omega$. Moreover, since all the $\lambda_{j}$ 's are different from zero, $S_{m}(x, t)$ must have mean value zero:

$$
\lim _{T \rightarrow \infty} \frac{1}{T} \int_{a}^{a+T} S_{m}(x, t) d t=0 .
$$

Hence, if $S_{m}(x, t)$ never changes sign it will have to be identically zero [4, Lemma $37_{9}$, p. 191]. On the other hand, if $S_{m}(x, t)$ changes sign once, due to almost periodicity it will have to change sign for an infinite sequence of pairs of values of $t$ which tend to $\infty$. In either case $S_{m}(x, t)$ will vanish for a sequence of values of $t$ which approach $\infty$. In view of (12), the same will then be true for $u(x, t)$ due to Rolle's theorem, and this completes the proof of Theorem 2 .

\section{REFERENCES}

1. R. Courant and D. Hilbert, Methods of mathematical physics. Vol. II, Interscience, New York, 1962. MR 25 \#4216.

2. V. A. Il'in, The solvability of mixed problems for hyperbolic and parabolic equations, Uspehi Mat. Nauk 15 (1960), no. 2 (92), 97-154=Russian Math. Surveys 15 (1960), no. 2, 85-142. MR 22 \#9721.

3. K. Kreith, Sturmian theorems for hyperbolic equations, Proc. Amer. Math. Soc. 22 (1969), 277-281. MR 39 \#5916.

4. N. Wiener, The Fourier integral and certain of its applications, Cambridge Univ. Press, Cambridge, 1933.

Department of Mathematics, Vanderbilt University, Nashville, Tennessee 37235 